\title{
Very thin transparent, conductive carbon nanotube films on flexible substrates
}

\author{
Vittorio Scardaci, ${ }^{1,2}$ Richard Coull, ${ }^{1}$ and Jonathan N. Coleman ${ }^{2,3, a)}$ \\ ${ }^{1}$ Hewlett-Packard DIMO, Liffey Park Technology Campus, Barnhall Road, Leixlip Co. Kildare, Ireland \\ ${ }^{2}$ Centre for Research on Adaptive Nanostructures and Nanodevices, Trinity College Dublin, Dublin 2, Ireland \\ ${ }^{3}$ School of Physics, Trinity College Dublin, Dublin 2, Ireland
}

(Received 18 January 2010; accepted 17 June 2010; published online 16 July 2010)

\begin{abstract}
We investigate the morphological, electrical, and optical properties of carbon nanotube thin films, focusing on films with transmittance, $T>90 \%$. For films with $T \approx 90 \%$ we measure sheet resistance of $R_{s}<400 \Omega / \square$. However, we show that optoelectrical properties, such as $\sigma_{\mathrm{dc}}$ and $\sigma_{\mathrm{dc}} / \sigma_{O p}$, degrade with decreasing film thickness, $t$, for percolating nanotube networks, i.e., those with $t$ $<20 \mathrm{~nm}$ and $T>90 \%$. Thus, while reducing $t$ can give $T>99 \%$, the corresponding $R_{s}$ increases to $>40 \mathrm{k} \Omega / \square$. Acid treatment improves the conductivity by doping, giving properties such as $T$ $\approx 98 \%$ for $R_{s} \approx 10 \mathrm{k} \Omega / \square$. ( ) 2010 American Institute of Physics. [doi:10.1063/1.3462317]
\end{abstract}

Transparent electrodes are essential components for devices such as displays, organic light emitting diodes, and solar cells. ${ }^{1}$ Indium tin oxide (ITO) is the most widespread material used for such applications. However, ITO has several drawbacks: its price has increased dramatically recently due to increasing demand while its brittleness makes it unsuitable for possible future flexible applications. ${ }^{2,3}$ Therefore, much research has been devoted to the development of alternative materials for ITO replacement. The minimum optical and electrical requirements for transparent electrodes are transmittance $T>90 \%$ and sheet resistance $R_{s}<100 \Omega / \square$. $T$ and $R_{S}$ are related by the following equation: ${ }^{4,5}$

$$
T=\left(1+\frac{Z_{0}}{2 R_{S}} \frac{\sigma_{O p}}{\sigma_{\mathrm{dc}}}\right)^{-2}
$$

where $Z_{0}=377 \Omega$ is the impedance of free space, and $\sigma_{O p}$ and $\sigma_{\mathrm{dc}}$ are the optical and dc conductivities, respectively. Hence, $T$ and $R_{s}$ are effectively controlled by $\sigma_{\mathrm{dc}} / \sigma_{O p}$. Thus, the minimum industry standard corresponds to $\sigma_{\mathrm{dc}} / \sigma_{O p}$ $>35$. Carbon nanotubes (CNTs) have been suggested as viable alternatives to ITO. ${ }^{5-10}$ At present, the highest $\sigma_{\mathrm{dc}} / \sigma_{O p}$ reported were $\sim 13$ for pristine CNT films ${ }^{9}$ and $\sim 15$ for composite films. ${ }^{10}$ This can be pushed further to $25-35$ by post-deposition acid treatment of pristine films. ${ }^{7,11}$ However, most of the data available in the literature are reported for relatively low $T$, usually below $85 \%$. Such films are typically above $40 \mathrm{~nm}$ in thickness and generally behave like bulk films. ${ }^{9}$ However, a number of applications, such as those in certain displays, require much higher transmittance values. Unfortunately, only limited data are available for highly transparent films. ${ }^{5-9}$ In addition, although it is usually assumed that $\sigma_{\mathrm{dc}}$ (and $\sigma_{\mathrm{dc}} / \sigma_{O p}$ ) is constant with thickness, recent experiments suggest that this is not the case and that, instead, it decreases for thin films. ${ }^{7,9}$ Thus, very thin films, with high $T$ values, are likely to have significantly reduced values of $\sigma_{\mathrm{dc}} / \sigma_{O p}$ and so much higher sheet resistances than expected. This is likely to be a significant problem for high $T$ applications. In this paper, we investigate the morphology and the optical and electrical properties of very thin CNT

${ }^{a)}$ Electronic mail: colemaj@tcd.ie. films, deposited by spray coating, with $T$ ranging from $70 \%$ up to $99 \%$.

Single wall CNTs, purchased from Iljin Nanotech, were dispersed in sodium dodecylsulphate by $5 \mathrm{~min}$ tip-sonication (VibraCell CVX, $750 \mathrm{~W}, 20 \% 60 \mathrm{kHz}$ ), then placed in a sonic bath (Model Ney Ultrasonic) for $1 \mathrm{~h}$ and tip-sonicated again for $5 \mathrm{~min}$. The dispersion was then centrifuged at 5500 rpm for $90 \mathrm{~min}$. The CNT concentration was determined on the top fraction of the centrifuged solution by absorption spectrophotometry. ${ }^{12}$ The solution was then diluted to 0.1 or $0.2 \mathrm{mg} / \mathrm{ml}$ and sprayed onto a polyethylene terephtalate (PET) substrate. The spray-coating process can be summarized as follows: PET, which had been previously degreased in acetone, was mounted onto a heated stage capable of moving in two dimensions relative to the spray source. CNTs were then sprayed by an atomising spray gun whose operation was linked to the raster movement of the stage. The sample is finally rinsed in water to partially remove the surfactant. After rinsing, some samples were treated with $12 \mathrm{M}$ nitric acid for $10 \mathrm{~min}$ to further improve the performance. This was followed by heat treatment at $100{ }^{\circ} \mathrm{C}$ for $2 \mathrm{~h}$ to remove doping effects introduced by acid treatment. Substrate temperature, stage scan speed, spray gun height, and spray flow rate were optimized to achieve the best film performance. Film thickness and transparency were controlled by CNT concentration and number of spray passes onto the substrate. The sheet resistance was measured by the four probe technique, using silver electrodes and a Keithley 2400 source meter. For uniformity measurements, transmission scans were carried out using a flat-bed Epson Perfection V700 Photo white light scanner at 150 dpi resolution. ${ }^{9}$ A transmittance map for every sample can be then achieved, where a single pixel corresponds to a single transmittance value. For electron microscopy, CNTs were sprayed, under the same conditions, onto glass coated by $\mathrm{Au} / \mathrm{Pd}$. Images were taken by a Zeiss Ultra plus field emission scanning electron microscope (SEM).

To investigate the morphology of our pristine CNT films, we carried out SEM analysis. Figures 1(a)-1(c) show typical SEM images of our films at low, medium, and high CNT density (low, medium, and high number of spray passes onto the substrate). It is clear that sparser networks are less uni- 


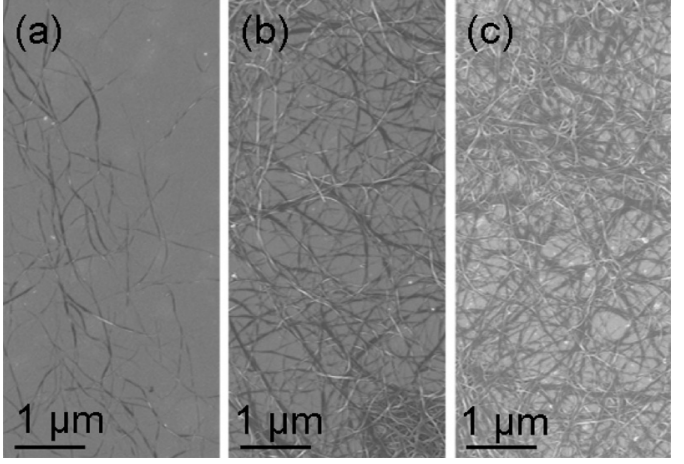

FIG. 1. [(a)-(c)] SEM images of representative CNT films at low, medium, and high number of spray passes, respectively.

form than dense ones. From statistical analysis carried out on SEM images across the samples, we derive average bundle sizes between 20 and $25 \mathrm{~nm}$, with no correlation with the number of spray passes.

Figure 2 shows typical $T$ versus $R_{S}$ plot of our results, with $T$ measured at $550 \mathrm{~nm}$. The dashed line in Fig. 2 represents the theoretical curve for $\sigma_{\mathrm{dc}} / \sigma_{O p}=11$. We can observe that films with $T \approx 90 \%$ display sheet resistances of $R_{s} \approx 400 \Omega / \square$. Such results are slightly inferior to those obtained by vacuum filtration ${ }^{9}$ but are consistent with those reported for the best spray cast pristine CNT films. ${ }^{7}$ However, data for $T>90 \%$ deviate significantly from the $\sigma_{\mathrm{dc}} / \sigma_{O p}=11$ curve, with the sheet resistance values much larger than those expected from Eq. (1). The thinnest film studied, that with $T \approx 99 \%$ (thickness, $t \approx 2 \mathrm{~nm}$ ), displayed a sheet resistance of $R_{s} \approx 40 \mathrm{k} \Omega / \square$, much larger than would be expected for $\sigma_{\mathrm{dc}} / \sigma_{O p}=11$.

This deviation becomes clearer if we use the $\left(T, R_{s}\right)$ data to calculate $\sigma_{\mathrm{dc}} / \sigma_{O p}$ using Eq. (1). We also calculate the average film thickness, $t$, from the $T$ data using: ${ }^{4}$

$$
T=\left(1+\frac{Z_{0}}{2} \sigma_{O p} t\right)^{-2},
$$

where we take $\sigma_{O p}=1.7 \times 10^{4} \mathrm{~S} / \mathrm{m}$ for pristine CNT films. ${ }^{9}$ We note that for thin films, $t$ becomes smaller than typical bundle diameters. In such cases, $t$ is an average over a sparse network and must be considered as a nominal thickness. Shown in Fig. 2 inset is a graph of $\sigma_{\mathrm{dc}} / \sigma_{O p}$ versus $t$. We see that $\sigma_{\mathrm{dc}} / \sigma_{O p}$ is only constant at $t>20 \mathrm{~nm}$ and falls off for

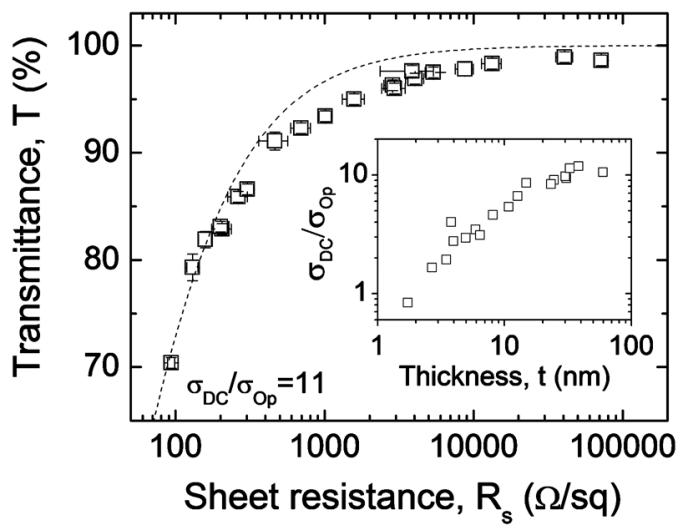

FIG. 2. Typical transmittance (taken at $550 \mathrm{~nm}$ ) vs sheet resistance plot for spray cast, pristine films. The dotted line represents $\sigma_{\mathrm{dc}} / \sigma_{O p}=11$. Inset: plot of $\sigma_{\mathrm{dc}} / \sigma_{O p}$ vs $t$. Note how $\sigma_{\mathrm{dc}} / \sigma_{O p}$ is thickness independent for $t>20$.

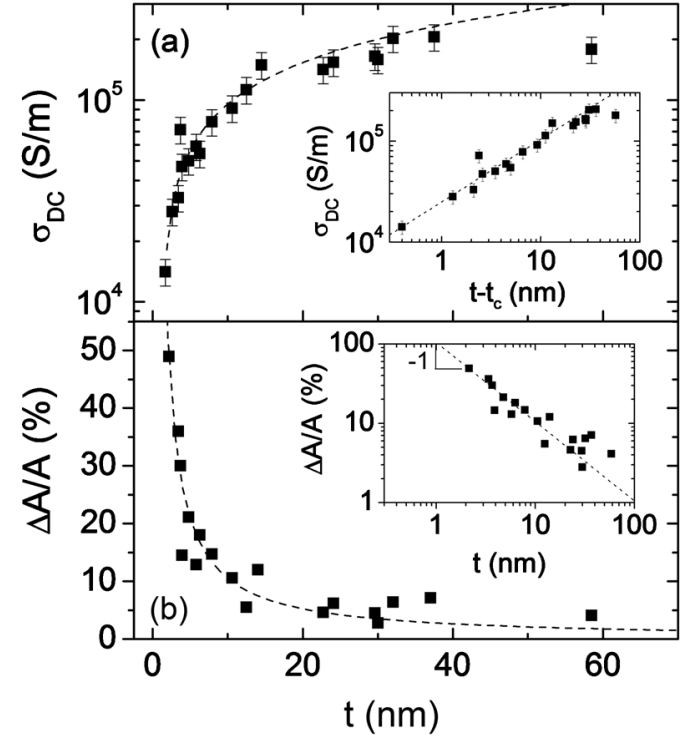

FIG. 3. (a) Plot of $\sigma_{\mathrm{dc}}$ as a function of nominal thickness, $t$. The trend is relatively flat for $t>20 \mathrm{~nm}$ while $\sigma_{\mathrm{dc}}$ decreases for $t<20 \mathrm{~nm}$ (the dotted line represents percolative behavior). Inset: data fitting according to the percolation equation. Note that the fit is poor for $t>20 \mathrm{~nm}$. (b) Thickness nonuniformity, $\Delta \mathrm{A} / \mathrm{A}$, as a function of mean film thickness, $t$. Inset: $\log -\log$ plot. Note that $\Delta \mathrm{A} / \mathrm{A}$ scales inversely with $t$ for $t<20 \mathrm{~nm}$. However, for $t>20 \mathrm{~nm}, \Delta \mathrm{A} / \mathrm{A}$ appears to flatten off.

lower $t$, reaching $\sigma_{\mathrm{dc}} / \sigma_{O P} \approx 1$ for $t \sim 2 \mathrm{~nm}(T \approx 99 \%)$. We suggest that this behavior is a manifestation of the changing behavior of the network morphology as the $t$ approaches the bundle size coupled with percolative effects at low $t$.

It is possible to improve the films by acid treatment which is known to remove the surfactant and introduce doping. ${ }^{7,8,11,13,14}$ After acid treatment, $R_{s}$ decreases to $\sim 30 \%$ of its original value. ${ }^{15}$ After dedoping by heat treatment, $R_{s}$ typically increased to $\sim 80 \%$ of its original value, suggesting that acid treatment works predominately by doping, with surfactant removal playing a minor role. The doping by acid and dedoping by heat treatment hypothesis was confirmed by optical measurements ${ }^{15}$ showing the $E_{s}{ }^{11}$ transitions disappear after acid treatment and reappear after heat treatment. ${ }^{13} \mathrm{We}$ note that after acid treatment, the $t=1.3 \mathrm{~nm}$ film had $R_{s}$ $\approx 10 \mathrm{k} \Omega / \square$ for $T \approx 98 \%$.

We calculate the dc conductivity from $\sigma_{\mathrm{dc}}=1 / R_{s} t$. Figure 3(a) shows that $\sigma_{\mathrm{dc}}$ is roughly constant at $\sim 2 \times 10^{5} \mathrm{~S} / \mathrm{m}$ for $t>20 \mathrm{~nm}$. Below this thickness, $\sigma_{\mathrm{dc}}$ decreases according to percolation theory as follows:

$$
\sigma_{\mathrm{dc}}=\sigma_{0}\left(t-t_{c}\right)^{\alpha},
$$

where $\sigma_{0}$ is a constant, $t_{c}$ the percolation threshold and $\alpha$ an exponent which is predicted to be 1.33 for two-dimensional networks. ${ }^{5,16}$ Fitting data in Fig. 3 by Eq. (3) yields $\sigma_{0}$ $=2.5 \times 10^{4} \mathrm{~S} / \mathrm{m}, \alpha=0.62$, and $t_{c}=1.3 \mathrm{~nm}$.

We map the local absorbance of the film, thus estimating local thickness nonuniformity, by carrying out transmission scanning. We refer to the ratio of the standard deviation of the absorbance to the measured (mean) absorbance, $\Delta \mathrm{A} / \mathrm{A}$, as the nonuniformity of the film. Figure 3(b) shows that nonuniformity is roughly constant for $t>20 \mathrm{~nm}$. However, for percolating networks $(t<20 \mathrm{~nm}) \Delta \mathrm{A} / \mathrm{A}$ increases inversely with $t$, indicating that $\Delta \mathrm{A}$ is thickness invariant.

It is important to note that $\sigma_{\mathrm{dc}} / \sigma_{O p}, \sigma_{\mathrm{dc}}$ and the thickness nonuniformity all demonstrate similar thickness depen- 
dences. All three parameters are relatively invariant for $t$ $>20 \mathrm{~nm}$. However, $\sigma_{\mathrm{dc}} / \sigma_{O p}$ and $\sigma_{\mathrm{dc}}$ decrease while $\Delta \mathrm{A} / \mathrm{A}$ increases as $t$ decreases below $t \approx 20 \mathrm{~nm}$. This behavior can be explained if we assume the films are bulk-like for $t$ $>20 \mathrm{~nm}$ but form a percolating network for $t<20 \mathrm{~nm}$. This degradation of performance with decreasing thickness will always be present for very thin films and will have important implications for nanotube networks in high $T$ applications.

In summary, we investigated a range of sprayed nanotube films with $70 \%<T<99 \%$. We find that $\sigma_{\mathrm{dc}} / \sigma_{O p}, \sigma_{\mathrm{dc}}$ and the thickness uniformity are not invariant with thickness for $t<20 \mathrm{~nm}(T>90 \%)$ but degrade at lower thicknesses due to percolation phenomena. For example, the dc to optical conductivity ratio falls from $\sigma_{\mathrm{dc}} / \sigma_{O p} \approx 11$ for thick films to $\sigma_{\mathrm{dc}} / \sigma_{O p} \approx 1$ for films with $T \approx 99 \%$. Extremely thin films displayed $T=99 \%$ and $R_{s} \approx 40 \mathrm{k} \Omega / \square$ which could be improved to $T>98 \%$ and $R_{s}<10 \mathrm{k} \Omega / \square$ on acid treatment.

We acknowledge the Science Foundation Ireland funded collaboration (SFI Grant No. 03/CE3/M406s1) between Trinity College Dublin and Hewlett Packard which has allowed this work to take place. J.N.C. is also supported by an SFI PI award.

${ }^{1}$ S. Watcharotone, D. A. Dikin, S. Stankovich, R. Piner, I. Jung, G. H. B. Dommett, G. Evmenenko, S. E. Wu, S. F. Chen, C. P. Liu, S. T. Nguyen, and R. S. Ruoff, Nano Lett. 7, 1888 (2007).
${ }^{2}$ Y. Leterrier, L. Medico, F. Demarco, J. A. E. Manson, U. Betz, M. F. Escola, M. K. Olsson, and F. Atamny, Thin Solid Films 460, 156 (2004).

${ }^{3}$ Z. Chen, B. Cotterell, and W. Wang, Eng. Fract. Mech. 69, 597 (2002).

${ }^{4} \mathrm{M}$. Dressel and G. Grèuner, Electrodynamics of solids: optical properties of electrons in matter (Cambridge University Press, Cambridge, 2002).

${ }^{5}$ L. Hu, D. Hecht, and G. Gruner, Nano Lett. 4, 2513 (2004).

${ }^{6}$ Z. C. Wu, Z. H. Chen, X. Du, J. M. Logan, J. Sippel, M. Nikolou, K. Kamaras, J. R. Reynolds, D. B. Tanner, A. F. Hebard, and A. G. Rinzler, Science 305, 1273 (2004).

${ }^{7}$ H. Z. Geng, K. K. Kim, K. P. So, Y. S. Lee, Y. Chang, and Y. H. Lee, J. Am. Chem. Soc. 129, 7758 (2007).

${ }^{8}$ H. Z. Geng, K. K. Kim, K. Lee, G. Y. Kim, H. K. Choi, D. S. Lee, K. H. An, Y. H. Lee, Y. Chang, Y. S. Lee, B. Kim, and Y. J. Lee, NANO 2, 157 (2007).

${ }^{9}$ E. Doherty, S. De, P. Lyons, A. Shmeliov, P. Nirmalraj, V. Scardaci, J. Joimel, W. Blau, J. Boland, and J. Coleman, Carbon 47, 2466 (2009).

${ }^{10}$ S. De, P. Lyons, S. Sorel, E. Doherty, P. King, W. Blau, P. Nirmalraj, J. Boland, V. Scardaci, J. Joimel, and J. Coleman, ACS Nano 3, 714 (2009).

${ }^{11}$ P. N. Nirmalraj, P. E. Lyons, S. De, J. N. Coleman, and J. J. Boland, Nano Lett. 9, 3890 (2009).

${ }^{12}$ S. Giordani, S. D. Bergin, V. Nicolosi, S. Lebedkin, M. M. Kappes, W. J. Blau, and J. N. Coleman, J. Phys. Chem. B 110, 15708 (2006).

${ }^{13}$ T. M. Barnes, J. L. Blackburn, J. van de Lagemaat, T. J. Coutts, and M. J. Heben, ACS Nano 2, 1968 (2008).

${ }^{14}$ J. L. Blackburn, T. M. Barnes, M. C. Beard, Y. H. Kim, R. C. Tenent, T. J. McDonald, B. To, T. J. Coutts, and M. J. Heben, ACS Nano 2, 1266 (2008).

${ }^{15}$ See supplementary material at http://dx.doi.org/10.1063/1.3462317 for effects of acid and heat treatment.

${ }^{16} \mathrm{D}$. Stauffer and A. Aharony, Introduction to Percolation Theory, 2nd ed (Taylor \& Francis, London, 1985). 